SLAC-PUB-11302

\title{
Stopping Gluinos
}

\author{
A. Arvanitaki ${ }^{1}$, S. Dimopoulos ${ }^{1 *}$, \\ A. Pierce ${ }^{2,1 \dagger}$, S. Rajendran ${ }^{1}$, and J. Wacker ${ }^{1}$ \\ ${ }^{1}$ Physics Department, Stanford University, \\ Stanford, California 94305, USA \\ 2 SLAC, Stanford University, \\ Menlo Park, California 94309, USA
}

\begin{abstract}
Long lived gluinos are the trademark of split susy. They form R-hadrons that, when charged, efficiently lose energy in matter via ionisation. Independent of R-spectroscopy and initial hadronization, a fraction of R-hadrons become charged while traversing a detector. This results in a large number of stopped gluinos at present and future detectors. For a $300 \mathrm{GeV}$ gluino, $10^{6}$ will stop each year in LHC detectors, while several hundred stop in detectors during Run II at the Tevatron. The subsequent decays of stopped gluinos produce distinctive depositions of energy in calorimeters with no activity in either the tracker or the muon chamber.
\end{abstract}

${ }^{*}$ The work of AA, SD, SR and JW is supported by National Science Foundation grant PHY-9870115 and the Stanford Institute for Theoretical, Physics

${ }^{\dagger}$ The work of AP is supported by the U.S. Department of Energy under contract 76SF00515.

Submitted to Physical Review D

Work supported by Department of Energy contract DE-AC02-76SF00515 


\section{Introduction}

The emergence of the landscape supports the idea that the smallness of the observed vacuum energy is explained by a fine-tuning. The hierarchy problem, historically the primary motivation for new weak-scale physics, might be solved by a similar fine-tuning. In this case, new physics is motivated by other considerations, such as gauge coupling unification and the existence of dark matter. Split superymmetry $[1,2,3]$ is such a concrete implementation of fine-tuned physics beyond the standard model, and will be tested at future colliders.

Split supersymmetry has a long lived gluino whose lifetime is determined by the extent to which the weak scale is fine-tuned. The gluino is the only new TeV-scale colored particle in split supersymmetry (SUSY), and therefore is the only particle copiously produced at hadronic accelerators. Discovering a long lived gluino is challenging because it typically leaves detectors without despositing a significant amount of its energy in detectors. Instead of decaying through a cascade of strong and electroweak transitions, the gluino may behave much like the lightest supersymmetric particle (LSP), revealing its presence through an excess of missing energy events. In this note we discuss a novel strategy for discovering the gluino: observing its decay after it has stopped within the detector.

If gluinos live longer than tens of nanoseconds, most pass through detectors without decaying. Gluinos that are stable on detector time scales have motivated several studies of split SUSY phenomenology $[4,5,6,7]$. There are also earlier studies where the gluino is the LSP or NLSP that have similar phenomenology $[8,9,10,11]$. While these searches can find a new, long-lived strongly interacting particle, they will not determine if this particle is absolutely stable. In-flight decays of the gluino result in displaced vertices, which indicate a finite gluino lifetime, but are only relevant for squark masses between $10^{2} \mathrm{TeV}$ and $10^{4} \mathrm{TeV}$ $[1,11,12]$. There have also been discussions of detecting nearly stable gluinos in cosmic ray showers [13] and if they were discovered in this fashion, would indicate very long gluino lifetimes.

In this letter, we point out that an observable fraction of gluinos may stop within a few meters of material, and thus within detectors ${ }^{1}$. This might allow observation of decays with much longer lifetimes - up to the running time of an experiment. For gluino masses greater than about $500 \mathrm{GeV}$, big bang nucelosynthesis constrains the gluino lifetime to be less than 100 seconds [15] and motivates searching for late decaying stopped gluinos. In concert with other "stable" gluino signatures, this method could play an important role in discovering split SUSY.

The organization of the paper is as follows. In Sec. 2 we discuss the production of gluinos at the Tevatron and the LHC. These long-lived gluinos hadronize upon production into "Rhadrons". In Sec. 3 we discuss the spectroscopy and relative production fraction of these particles. Even if the majority of R-hadrons are born neutral, we show that a population of charged R-hadrons is induced by interactions within the detector. In Sec. 4 we discuss how this occurs, as we study the propagation of the R-hadrons through matter. We then compute the number of stopped R-hadrons as a function of distance in iron. This allows an estimate of the number of stopped gluinos in current and future detectors (Sec. 5). When a

\footnotetext{
${ }^{1}$ This is analogous to an idea studied recently [14] for stopping slepton "next-to lightest supersymmetric particles" (NLSPs) and examining their late decays.
} 


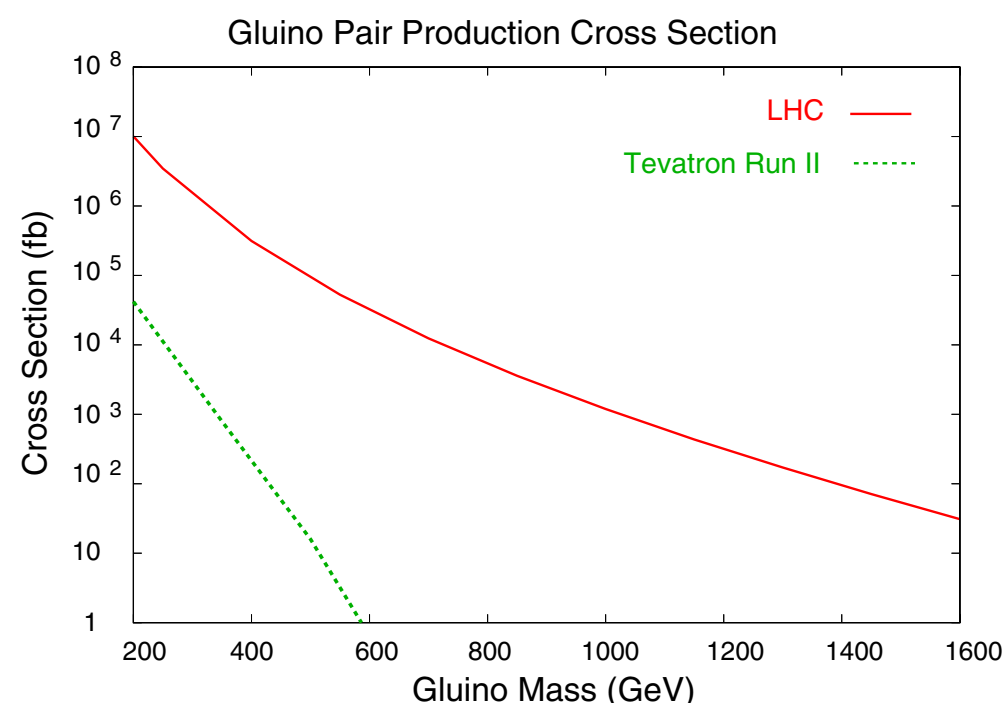

Figure 1: The gluino production cross section as a function of mass at the LHC (red solid) and Tevatron Run II (green dashed).

gluino ultimately decays, it will give rise to jets, not originating from the primary interaction point but from its stopping point. A detector will observe out-of-time energy deposition in the calorimeters which will appear as missing energy relative to the interaction region. We discuss these features in Sec. 5.1.

\section{Gluino Production}

We calculate the production rate of gluinos at both the LHC $(\sqrt{s}=14 \mathrm{TeV})$ and the Tevatron $(\sqrt{s}=1.96 \mathrm{TeV})$ using the CTEQ4l parton distribution functions (PDFs) [17]. To take into account the enhancement found at next-to-leading order(NLO), we evaluate the leading order expression (see, e.g., [18]) at $Q^{2}=\left(0.2 m_{\tilde{g}}\right)^{2}$, where the leading-order and NLO results match [19]. At low masses, the gluino production rate at the LHC is extraordinary, reaching $\sim 1 /$ sec for $m_{\tilde{g}} \approx 350 \mathrm{GeV}$. At low velocities, the Sommerfeld resummation of the "Coulomb ladder" gives an $\pi \alpha_{s} / v$ enhancement for the production of slow gluinos. This is particularly relevant for the $g g \rightarrow \tilde{g} \tilde{g}$ subprocess where the gluons are in an attractive state. We model the Sommerfeld enhancement by multiplying the cross section for the $g g \rightarrow \tilde{g} \tilde{g}$ subprocess by

$$
E_{s}=\frac{C \pi \alpha_{s} / v}{1-\exp \left(-C \pi \alpha_{s} / v\right)},
$$

with $C=1 / 2[9,16]$. This coefficient comes from a color-averaging of the various initial and final states. Most of the difference between the leading order and NLO production cross sections arises from physics at distance scales much shorter than those responsible for the Sommerfeld enhancement. So, it seems reasonable to treat these two contributions as 

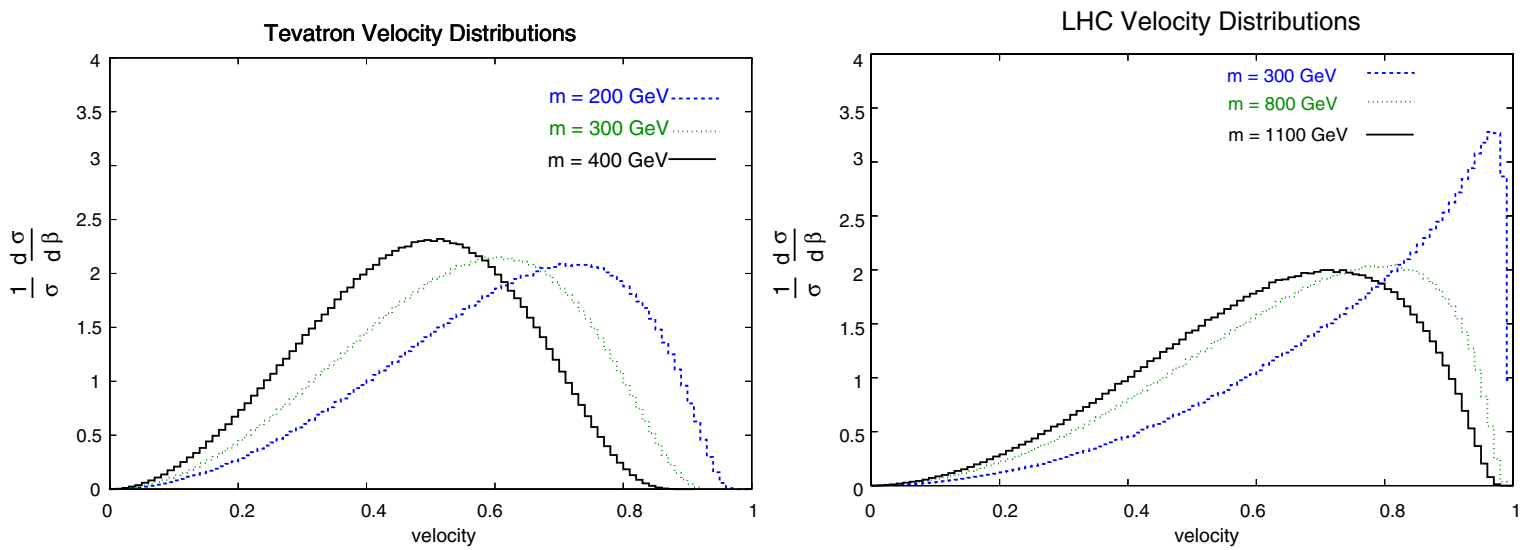

Figure 2: The distribution of gluino velocities at the LHC (right) and Tevatron (left). In each case we have shown the distribution for multiple gluino masses. At the Tevatron, we show $m_{\tilde{g}}=200,300,400 \mathrm{GeV}$ as dashed blue, dotted green, and solid black, respectively. At the LHC, we show the distribution for $m_{\tilde{g}}=300,800,1100 \mathrm{GeV}$ as dashed blue, dotted green, and solid black, respectively.

factorizable. As an approximation, we take:

$$
\sigma=E_{s} \times\left.\sigma_{L O}\right|_{\mu=0.2 m_{\tilde{g}}}
$$

The integrated cross sections for gluino pair production at the Tevatron and the LHC are shown in in Fig. 1. We have placed the most minimal of cuts, $\left|\eta_{\tilde{g}}\right|<4$.

While the cross section for the gluino production is a steeply falling function of the gluino mass, the number of slowly moving gluinos does not fall quite as steeply. This is because the velocity distribution skews toward smaller velocities as the mass of the gluino increases. We show the normalized velocity distribution in Fig. 2. Even for the lightest masses we consider at the Tevatron, $m_{\tilde{g}}=200 \mathrm{GeV}$, the gluino is produced with non-relativistic velocities. In contrast, a $300 \mathrm{GeV}$ gluino at the LHC produced relativistically. Gluinos at the LHC do not become non-relativistic until masses around $1 \mathrm{TeV}$.

These distributions change as a function of the pseudo-rapidity, $\eta$. There are fast gluinos in the forward region due to a boost going from the parton center of mass frame to the lab frame. This trend can be seen in Fig. 3 for a $300 \mathrm{GeV}$ gluino at the LHC. While somewhat moderated at higher masses, the trend persists. Since only the slowest gluinos will stop, the stopped gluinos will preferentially be in the central part of the detector. We revisit this point in Sec. 5 .

\section{Spectroscopy and Hadronization}

After production, gluinos combine with light degrees of freedom to form colorless hadrons. The mass spectrum of these hadrons will affect the properties of gluinos propagating through the detector. We do not compute the spectrum of the R-hadrons from first principles. Rather, 


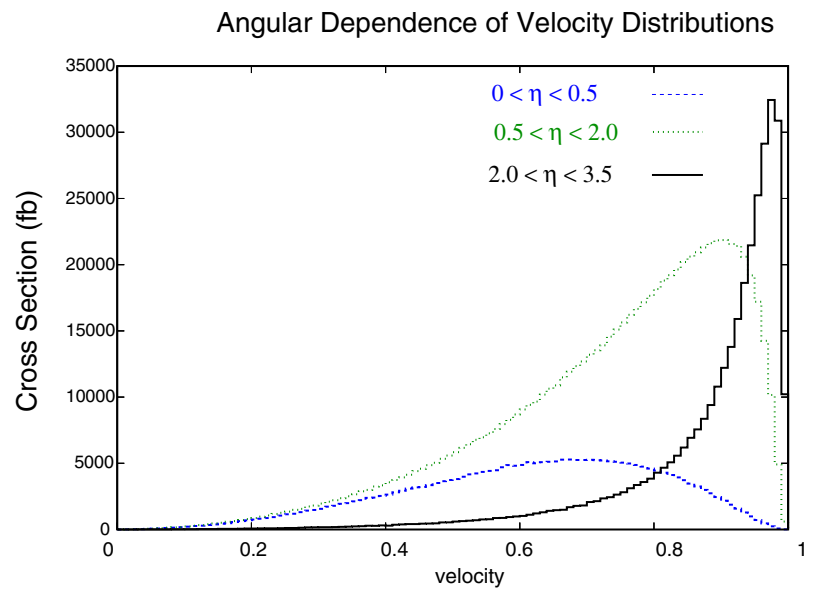

Figure 3: The distribution of gluino velocities at the LHC for $m_{\tilde{g}}=300 \mathrm{GeV}$ as a function of rapidity. Shown are curves for $0<|\eta|<0.5$ (dashed blue ), $0.5<|\eta|<2.0$ (dotted green), $2<|\eta|<3.5$ (solid black).

we enumerate the relevant possibilities, and look at the consequences of each. Electromagnetic interactions will be the dominant mechanism for R-hadrons to stop. We therefore pay particular attention to whether there are long lived charged R-hadrons.

We will treat isospin as a good symmetry since the dominant breaking arises through light quark masses and electromagnetic effects. The splittings in the isospin multiplet are a few $\mathrm{MeV}$ and for the energies and processes we are considering, these splittings are sufficiently small to be irrelevant. For simplicity, we exclude the possibility of strange valence quarks. Including them would not significantly affect the results of this paper.

\section{Mesons}

R-mesons can be $(\tilde{g} g)$ or $(\tilde{g} q \bar{q})$ states. The $(\tilde{g} q \bar{q})$ states can be classified by their isospin. Considering only $u$ and $d$ quarks, there is an isosinglet and an isotriplet.

The R-meson spectroscopy is the most uncertain because we know very little about the constituent mass of a gluon from QCD. The constituent mass of the gluon may be

estimated as $700 \mathrm{MeV}$, but there are large uncertainties. Because it is roughly about twice the constituent mass of the a light quark, the mass ordering of a gluino-gluon state $\left(R_{g}\right)$ and the gluino-quark-anti-quark state $\left(R_{q \bar{q}}\right)$ is unclear. Moreover, the isosinglet $R_{q \bar{q}}$ will mix with $R_{g}$ and the distinction between these two states is artificial.

The isotriplet possesses charged states which will efficiently lose energy, so it is important to determine how often the gluino is in the isotriplet, and how often it is in an isosinglet state. Thus, the most important aspect of R-meson spectroscopy is the mass of the lightest isotriplet state relative to the lightest isosinglet state. We denote this mass difference by $\Delta M_{31} \equiv M_{\mathbf{3}}-M_{\mathbf{1}}$.

There are three distinct possibilities for $\Delta M_{31}$ :

- Mass Region 1: $\Delta M_{31}>m_{\pi} \quad$ Isosinglet is long lived. 
- Mass Region 2: $\left|\Delta M_{31}\right|<m_{\pi}$

- Mass Region 3: $\Delta M_{31}<-m_{\pi}$
Both are long lived.

Isotriplet is long lived.

In the first and last case, the heavier meson will be unstable to strong decays. Thus, all mesons exiting the interaction region will either be isosinglets or isotriplets, respectively. In Mass Region 1, all R-mesons exiting the interaction region will be neutral. In Mass Region 3, one third will be charged. These represent two extreme cases; the middle region interpolates between the two. As we will see, the final results for stopping will often be similar even for Regions 1 and 3, indicating insensitivity to the spectroscopy.

If the mass splitting $\left|\Delta M_{31}\right|$ is less than $m_{\pi}$, the only way for the heavier state to decay is through a weak transition. Weak decays that change the spin by one unit have a mean lifetime of

$$
\tau_{\Delta j=1}=\tau_{n}\left(\frac{\Delta M_{n p}}{\Delta M_{31}}\right)^{5} \sim 60 \mathrm{~ns}\left(\frac{130 \mathrm{MeV}}{\Delta M_{31}}\right)^{5},
$$

where $\tau_{n}$ is the neutron lifetime and $\Delta M_{n p}$ is the proton-neutron mass difference. This lifetime is of the order of the stopping time of the R-hadron and therefore is not important qualitatively. In particular, if the phase space is even somewhat small, then this effect can be completely neglected. Decays will occur after a gluino has stopped. A decay will give the gluino a tiny kick, but the velocity will be sufficiently small that the gluino will rapidly come to rest again.

In Mass Region 2 both the isosinglets and isotriplets live long enough that we must consider how both propagate through the detector and make assumptions about their initial production fraction. The produced ratio of isotriplet to isosinglet R-hadrons exclusively involving light quarks is easy to estimate: $N_{R_{3}}: N_{R_{1}}=3: 1$. The main uncertainty in hadronization is the number of $R_{g}$ that are made and how light they are relative to the $R_{q \bar{q}}$ isosinglet. There is no reason to expect $R_{g}$ production to completely dominate the production of the isotriplet state.

\section{Baryons}

The next question is whether there are long-lived charged R-baryons ( $\tilde{g} q q q)$. Since splittings within an isomultiplet are small, this question is equivalent to whether the lightest baryonic state is an isosinglet or a larger isomultiplet.

It seems unlikely that the lightest R-baryon is an isosinglet ( $\tilde{g} u d s$ ) because it requires the inclusion of a strange quark, costing an extra $\sim 150 \mathrm{MeV}$. While this possibility can not be excluded completely, we temporarily ignore it, and revisit its consequences in the conclusion. The remaining possibilities are that either an isodoublet or isoquartet is the lightest baryonic multiplet. The isoquartet has an intruiging doubly charged state, but for simplicity we will assume the isodoublet is the lightest. The conclusions related to stopping are insensitive to this assumption.

The most important spectroscopic feature is the difference in mass between an R-meson and an R-baryon. If the inequality $M_{R_{M}}+m_{N}>M_{R_{B}}+m_{\pi}$ is satisfied, then there are 
exothermic conversions of R-mesons to R-baryons as the R-hadrons propagate through matter (see Sec. 4). This seems very likely since the pion is anomalously light due to its pseudoGoldstone nature. We will assume this inequality throughout the paper. Because there is no strange matter in the detector, matter conversion will only produce isodoublet R-baryons and not isosinglets.

Roughly only $\mathcal{O}(1 \%)$ of the R-hadrons produced directly will be R-baryons or R-antibaryons[6]. As we will discuss in the next section, the dominant process for producing slow R-baryons is conversion of R-mesons in matter, rendering the uncertainty in this initial hadronization fraction largely irrelevant for determining the fraction of stopped gluinos.

\section{Propagation Through Matter}

When the R-hadrons are charged, the dominant energy loss is through ionization as described by the Bethe-Bloch equation. Neutral R-hadrons will not slow appreciably because they have huge momentum and no long range forces. We will discuss several interactions important for determining the fraction of the time that the R-hadrons are charged.

\subsection{Bethe-Bloch}

Energy loss via ionization will effectively stop charged particles that are moving with $\beta \gamma \lesssim 1$. As discussed in Sec. 2, a reasonable fraction of the R-hadrons are in this regime, particularly in the central region. Thus, the crucial question will be what fraction of the propagation distance is the particle charged.

The Bethe-Bloch formula for the rate of energy loss in matter is:

$$
\frac{d E}{d x}=-\frac{4 \pi \alpha^{2} \rho}{A m_{p} m_{e}} \frac{Z z^{2}}{v^{2}}\left(\frac{1}{2} \ln \frac{2 m_{e} v^{2}}{I\left(1-v^{2}\right)}-v^{2}\right),
$$

where $E$ is the energy of the incident particle; $A$ and $Z$ are the atomic mass and number of the absorber, respectively; $\rho$ is the mass density of the material; $z$ is the charge of the incident particle; and $m_{e}$ and $m_{p}$ are the masses of the electron and proton respectively. In the non-relativistic limit, $E \simeq m_{\tilde{g}}+\frac{1}{2} m_{\tilde{g}} v^{2}$, and the Bethe-Bloch equation can be recast in the form

$$
\frac{d v}{d x}=-\frac{1}{x_{0} v^{3}}\left(1+\frac{\log v}{\kappa}+\mathcal{O}\left(v^{2}\right)\right)
$$

where $x_{0}$ and $\kappa$ are material dependent. Parametrically these two constants are given by

$$
x_{0} \sim \frac{1}{\alpha m_{e}} \frac{m_{\tilde{g}}}{m_{e}} \frac{1}{4 \pi Z \alpha^{4} \log \alpha^{-1}} \quad \kappa \sim \log \alpha^{-1} .
$$

For iron, setting $m_{\tilde{g}}=500 \mathrm{GeV}$, these are $x_{0}=526 \mathrm{~m}$ and $\kappa=4.23$ (see Table 1 for other materials).

Insight into the approximate stopping distance can be found by dropping the $\log v$ term in the Bethe-Bloch formula. Integration yields:

$$
x=\frac{x_{0} v^{4}}{4}\left(\frac{m_{\tilde{g}}}{500 \mathrm{GeV}}\right) .
$$




\begin{tabular}{|c|c|c|}
\hline & $x_{0}$ & $\kappa$ \\
\hline \hline Iron & $526 \mathrm{~m}$ & 4.23 \\
Lead & $503 \mathrm{~m}$ & 3.60 \\
Uranium & $313 \mathrm{~m}$ & 3.53 \\
Copper & $491 \mathrm{~m}$ & 4.10 \\
\hline
\end{tabular}

Table 1: Coefficients of the Bethe-Bloch equation for common materials in detectors for $m_{\tilde{g}}=500 \mathrm{GeV}[20]$. See Equation 5 .

Thus, given a fixed length $x$ of material, all particles with velocities beneath

$$
v \lesssim\left(\frac{4 x}{x_{0}}\right)^{\frac{1}{4}}\left(\frac{500 \mathrm{GeV}}{m_{\tilde{g}}}\right)^{\frac{1}{4}}
$$

will stop. Most detectors have the equivalent of one to two meters of iron in the radial direction; therefore, $500 \mathrm{GeV}$ gluinos will stop in detectors if their velocities are less than $v \sim 0.30-0.35$.

The Bethe-Bloch formula breaks down for velocities beneath the velocity where ionization reaches its maximum, $v_{\max }$ ion. This velocity is given by $v_{\max }$ ion $\simeq \exp \left(-\kappa+\frac{1}{3}\right)$. Since $\kappa$ is parametrically $\mathcal{O}\left(\log \alpha^{-1}\right), v_{\text {max ion. }} \sim \mathcal{O}(\alpha)$. Physically, this when the incident particle becomes an adiabatic perturbation on the electrons in an atom. For iron, $v_{\max }$ ion. $=0.015$. Stopping below this velocity can be described by the Fermi-Teller theory [21]. In FermiTeller, $d v / d x$ is constant for velocities beneath $v_{\max }$ ion. This theory was later extended by of Lindhard and Scharff[22]. We approximate the post-Bethe-Bloch region beginning at $v_{\text {max ion. }}$ In this approximation the stopping distance beneath this velocity is

$$
\Delta x=3 \kappa x_{0} v_{\max \text { ion. }}^{4} \sim \frac{m_{\tilde{g}}}{\alpha^{2} m_{e}^{2}} \sim 1 \mathrm{~cm} .
$$

Extrapolating from the data of muons stopping on copper, the stopping distance for a 500 $\mathrm{GeV}$ gluino after the break down of the Bethe-Bloch formula is $\Delta x=0.25 \mathrm{~cm}$. This is on par with the above estimate.

\subsection{Matter Conversion}

Processes may be catalyzed by the propagation of the gluino through matter. Interactions of the gluino with light degrees of freedom are inefficient at slowing the gluino. In analogy to a bowling ball moving through a sea of ping-pong balls, the kinematics prevent any appreciable momentum loss by the incident gluino. However, these interactions can have an important indirect effect-they can cause an uncharged R-hadron to acquire charge. The charged particle can then efficiently lose energy via ionization. We will therefore treat these interactions as "label-changing" events.

\section{Meson to Baryon Conversion}

We consider a process in which $R$-mesons can interact with baryons in the detector to become R-baryons: $R_{m}+$ Baryon $\rightarrow R_{b}+\pi$ [6]. As mentioned in Sec. 3, this process is likely 


\begin{tabular}{|c|c|}
\hline & $\ell$ \\
\hline \hline Copper & $0.15 \mathrm{~m}$ \\
Iron & $0.17 \mathrm{~m}$ \\
Lead & $0.17 \mathrm{~m}$ \\
Uranium & $0.11 \mathrm{~m}$ \\
\hline
\end{tabular}

Table 2: Nuclear interaction lengths for common material in calorimeters.

exothermic because the pion is an anomalously light meson as it is pseudo-Goldstone boson.

Exothermic reactions have enhanced cross sections at low velocities. This is derivable from Fermi's golden rule:

$$
\sigma v_{\text {rel }} \propto|\mathcal{M}|^{2} \frac{p_{f}^{2}}{v_{f}},
$$

where $|\mathcal{M}|^{2}$ is the matrix element for the transition, $v_{\text {rel }}$ is the relative velocity of the incoming state and $p_{f}^{2} / v_{f}$ is the result of the final-state density of states. For the case when there is a light outgoing particle, the density of states simplifies to $m_{f}^{2} v_{f}$. The cross section then goes as

$$
\sigma=\sigma_{0} \frac{v_{f}}{v_{\text {rel }}}
$$

For exothermic processes, $v_{f}$ is fixed; therefore as $v_{\text {rel }} \rightarrow 0$ the cross section increases.

Thus, at small velocities meson to baryon conversion is enhanced. After a short distance, the R-mesons are depleted and transform into isodoublet R-baryons. The exothermic nature of this interaction also ensures that once R-hadrons are converted to R-baryons, they will not revert to R-mesons. As we will see, the low-velocity enhancement will make our results largely insensitive to assumptions about spectroscopy and cross sections.

To parameterize our ignorance about the strong interactions, we consider three different conversion cross sections, $\sigma_{0}=30 \mathrm{mb}, 3 \mathrm{mb}, 0.3 \mathrm{mb}$. In all cases, the cross sections will be enhanced at low velocities by the ratio $v_{\pi} / v_{\text {rel }}$, where $v_{\pi}$ is the velocity of the outgoing pion. R-meson to R-baryon conversion should release several hundred $\mathrm{MeV}$ of energy $(Q \approx 400$ $\mathrm{MeV}$ ), which ensuring that the outgoing pions are relativistic. We set $v_{\pi} \simeq 1$ from now on.

The R-meson will scatter off nucleons confined within a nucleus. These nucleons are not at rest with respect to the lab frame, and have a Fermi velocity $v_{F}$. The average binding energy per nucleon in a nucleus is $\mathcal{O}(8 \mathrm{MeV})$ which means that the Fermi velocity is $v_{F} \simeq 0.15$. For scatterings with an incident velocity less than the Fermi velocity, $v_{\text {rel }} \approx v_{F}$, while for incident velocities faster than $v_{F}$ the incident velocity, $v_{\text {rel }} \approx v_{\text {inc }}$.

The interaction lengths in iron for each of the three benchmark conversion cross sections are

$$
\ell_{\mathrm{Fe}}^{30 \mathrm{mb}}=0.17 \mathrm{~m} v_{\mathrm{rel}} \quad \ell_{\mathrm{Fe}}^{3 \mathrm{mb}}=1.7 \mathrm{~m} v_{\mathrm{rel}} \quad \ell_{\mathrm{Fe}}^{0.3 \mathrm{mb}}=17 \mathrm{~m} v_{\text {rel }} .
$$

Note, the $30 \mathrm{mb}$ interaction length corresponds to the interaction length of an ordinary pion in material. In Table 2 we give the nuclear interaction lengths for other common materials 
in detectors. For the first two cases, the majority of slow R-mesons are depleted by the end of a few meters, while even for the smallest cross section, a reasonable fraction of the slowest R-mesons are converted at the end of a typical detector. Only slow R-hadrons have a chance of stopping in a detector since the stopping distance goes as $x_{\text {stop }} \sim x_{0} v^{4}$ with $x_{0} \sim 500 \mathrm{~m}$. Therefore, the R-mesons that are capable of stopping are converted to Rbaryons. This insulates us from the details of spectroscopy and even makes the distinction between a conversion cross section of $30 \mathrm{mb}$ and $3 \mathrm{mb}$ small. In fact, the only situation which will be appreciably different than the others is when the isosinglet R-meson is significantly lighter than the isotriplet and the conversion cross section is anomalously small.

$\mathrm{R}$-meson to R-baryon conversion is the dominant process for slow R-baryon production. Only isodoublet baryons are produced in this process. We assume that the isosinglet baryon is not several hundred $\mathrm{MeV}$ lighter than the isodoublet baryons, so that the isodoublet baryons are long-lived. Since the isodoublet has a charged state, these stop efficiently. A fraction of the R-mesons will thus be converted early to R-baryons and then have sufficient time to stop.

\section{Charge Oscillation}

Pion exchange will switch the R-hadrons between the states of an isomultiplet. The cross section for this process should be comparable to those of strong interactions (30 mb) but could be somewhat smaller due to the octet nature of the light quark cloud around the gluino. This uncertainty does not significantly affect our results, so we leave the cross section at 30 mb throughout for simplicity. With a $30 \mathrm{mb}$ cross section, the interaction length is much shorter than the detector. Charge oscillation can therefore be approximated by a R-hadron that spends a fraction of its life charged. A doublet R-baryon will spend half its time charged, while the isotriplet R-meson will spend two-thirds of its transit time charged. During the time they are charged, the R-hadrons undergo Bethe-Bloch deceleration, and while they are neutral, they propagate freely until their next charge exchange process.

In Mass Region $2\left(\left|\Delta M_{31}\right|<m_{\pi}\right)$ both the isotriplet and isosinglet are long lived. The isosinglet can become an isotriplet and vice versa by emitting a pion. We again estimate that this cross section is $30 \mathrm{mb}$. One of these transitions is exothermic and becomes important at low velocities, while the reverse process will be endothermic and will turn off at low velocities. When the isosinglets are heavier than the isotriplet they are depleted and the remaining isotriplets will spend $\frac{2}{3}$ of the their time charged. This means that they will stop more quickly than R-baryons, which only spend one half their time charged. So, in the case where the isotriplet is light, a greater number of R-hadrons can stop in a detector if the R-meson to R-baryon cross section is small.

\subsection{Nuclear Capture}

After R-hadrons have slowed significantly, they may be captured by a heavy nucleus. We view this process as the likely final state for a stopping gluino. Thus even if a stopped charged R-hadron transitions to a neutral state, it does not "wander off" and is really trapped.

Nuclei capture all hadronic particles provided they satisfy the following criteria for absorption. First, the incident particle should not transfer too much momentum in a single 
collision to kick out a nucleon from the absorber. Second, the momentum transfer should be small enough so that the incident particle couples coherently to an entire nucleon rather than individual quarks, $\delta q \lesssim \Lambda_{\mathrm{QCD}}$. Finally, in the center of momentum frame, both particles must come to rest. In the case we consider here, the kinematics are different than typical nuclear physics processes where a heavy nucleus absorbs a light incident particle. Nuclear capture of R-hadrons is near the opposite limit: the absorbing nucleus is the light object and in the center of momentum frame the R-hadron is nearly stationary. The condition for capture is that the nucleus must come to rest.

Particles that have slowed down sufficiently to be captured are charged and therefore have isospin. Thus the stopping R-hadron likely couples to pions, and we estimate $\sigma \sim 1 / \Lambda_{\mathrm{QCD}}^{2}$. We now consider whether the above criteria are satisfied. The number of interactions in a nucleus scales as $N_{\text {int }} \sim A^{\frac{1}{3}}$. The condition for absorption becomes

$$
\Delta q \sim N_{\text {int }} \delta q=A^{\frac{1}{3}} m_{n} v_{F} \gtrsim A m_{n} v
$$

where $v_{F}$ is the average Fermi velocity of nucleons within the nucleus. Now the condition for binding becomes $v \lesssim A^{-\frac{2}{3}} v_{F}$. This shows that at slow enough velocities R-hadrons are captured. The total momentum transfer necessary for the R-hadron to be absorbed is smaller in lighter nuclei which shows that they are better absorbers of R-hadrons.

\section{$5 \quad$ Stopped Gluinos}

To calculate the number of R-hadrons stopped at CDF, D0, ATLAS, and CMS, we utilize the initial velocity distributions from Sec. 2 and incorporate the interactions from Sec. 4. We also characterize how the stopped gluinos are distributed throughout the detectors. All the presented results will be for Mass Region 1, where the lightest R-meson is an isosinglet. This represents a "worst-case scenario", where stopping is due solely to conversion to R-baryons. If there are stable charged mesons, as in Mass Region 3, these too would slow down via ionization losses, leading to more stopped R-hadrons.

We consider three cases in this section corresponding to R-meson to R-baryon conversion cross sections of $\sigma_{0}=30,3,0.3 \mathrm{mb}$. There is little difference between the two higher cross sections. For either of these two cross sections, R-mesons rapidly convert to R-baryons at the low velocities that are important for stopping.

To estimate the total number of particles stopped, we must estimate the total amount of material in each detector. As a first approximation we present the number stopped in 2 meters of iron in Fig. 4. The difference between the $30 \mathrm{mb}$ and $3 \mathrm{mb}$ conversion cross sections are relatively small. Decreasing the cross section further does make a difference, however. The lowest cross section, $\sigma_{0}=0.3 \mathrm{mb}$, results in an order of magnitude fewer stopped R-hadrons after 2 meters.

Making a more sophisticated estimate of the number stopped in various detectors requires taking into account the detector geometries and their different compositions. The four main detectors have roughly the following coverage and material depths:

- CDF's EM calorimeter contains $16 \mathrm{~cm}$ of $\mathrm{Pb}$ in the radial direction. The hadronic calorimeter contains $77 \mathrm{~cm}$ of Fe. Each calorimeter covers $|\eta|<3.6$. 


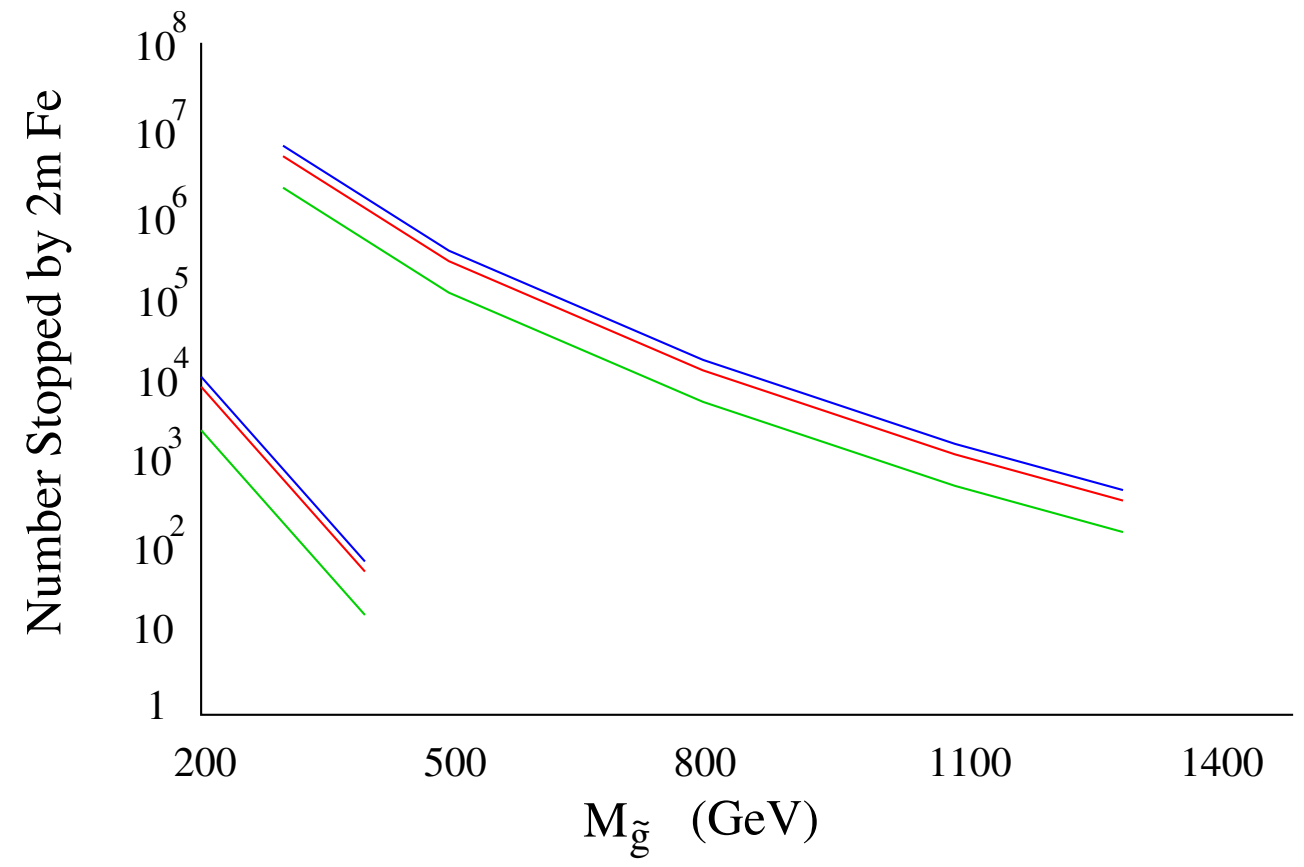

Figure 4: The number of R-hadrons stopped after two meters of iron in Mass Region 1. This plot convolutes the velocity distribution at production with conversion processes and matter and ionization losses. The upper set of curves is for the LHC for a total accumulated luminosity of $100 \mathrm{fb}^{-1}$, equivalent to a year of running at high luminosity. The lower set is for the Tevatron Run II, assuming a total of $2 \mathrm{fb}^{-1}$. In each set the curves correspond to a meson to baryon conversion cross section, $\sigma_{0}=30 \mathrm{mb}, 3 \mathrm{mb}$, and $0.3 \mathrm{mb}$ from top to bottom.

- D0's EM calorimeter contains about $7 \mathrm{~cm}$ of U; the fine hadronic calorimeter has 35 $\mathrm{cm}$ of $\mathrm{U}$, while the coarse hadronic calorimeter has $48 \mathrm{~cm}$ of $\mathrm{Cu}$, all with coverage out to $|\eta| \lesssim 4$.

- ATLAS's EM calorimeter has $66 \mathrm{~cm}$ of $\mathrm{Pb}$, while its hadronic calorimeter contains $156 \mathrm{~cm}$ of Fe. Both calorimeters cover up to $|\eta|<3.2$.

- CMS's EM calorimeter has $46 \mathrm{~cm}$ of Pb, while its hadronic calorimeter contains $98 \mathrm{~cm}$ of $\mathrm{Cu}$, both cover up to $|\eta|<3$.

Taking into account the amount of absorber in the each detector, we estimate the number of gluinos stopped in Table 3. We take the meson to baryon cross conversion cross section to be $\sigma_{0}=3 \mathrm{mb}$. The number stopped can be substantial; for instance a $300 \mathrm{GeV}$ gluino, $\sim 10^{6}$ should stop in each LHC detector in a year of high luminosity $\left(100 \mathrm{fb}^{-1}\right)$ running. At the Tevatron, hundreds of $300 \mathrm{GeV}$ gluinos stop in each detector after $2 \mathrm{fb}^{-1}$ of running.

We now consider the distribution of stopped gluinos within the detector. In Fig. 5, we plot the stopping profiles at both the Tevatron and the LHC for propagation through a hypothetical iron detector. Curves for different meson to baryon conversion cross sections 


\begin{tabular}{|c||c|c|c|}
\hline $2 \mathrm{fb}^{-1}$ & $200 \mathrm{GeV}$ & $300 \mathrm{GeV}$ & $400 \mathrm{GeV}$ \\
\hline $\mathrm{CDF}$ & $4.1 \times 10^{3}$ & $3.1 \times 10^{2}$ & $3.3 \times 10^{1}$ \\
$\mathrm{D} 0$ & $4.5 \times 10^{3}$ & $3.3 \times 10^{2}$ & $3.4 \times 10^{1}$ \\
\hline $100 \mathrm{fb}^{-1}$ & $300 \mathrm{GeV}$ & $800 \mathrm{GeV}$ & $1300 \mathrm{GeV}$ \\
\hline ATLAS & $5.8 \times 10^{6}$ & $1.8 \times 10^{4}$ & $6.2 \times 10^{2}$ \\
$\mathrm{CMS}$ & $3.7 \times 10^{6}$ & $1.2 \times 10^{4}$ & $3.9 \times 10^{2}$ \\
\hline
\end{tabular}

Table 3: The estimated total number of gluinos stopped in each detector for a $3 \mathrm{mb}$ R-meson to R-baryon conversion cross section.

are plotted in this figure. Larger conversion cross sections allow for more rapid stopping. As can be seen in the figure, for the larger two cross-sections, essentially all slow mesons have a chance to convert to baryons. Therefore, a a similar fraction stop by 1 meter. For the smallest conversion cross section, not all mesons have converted, and the total number stopped is substantially fewer.
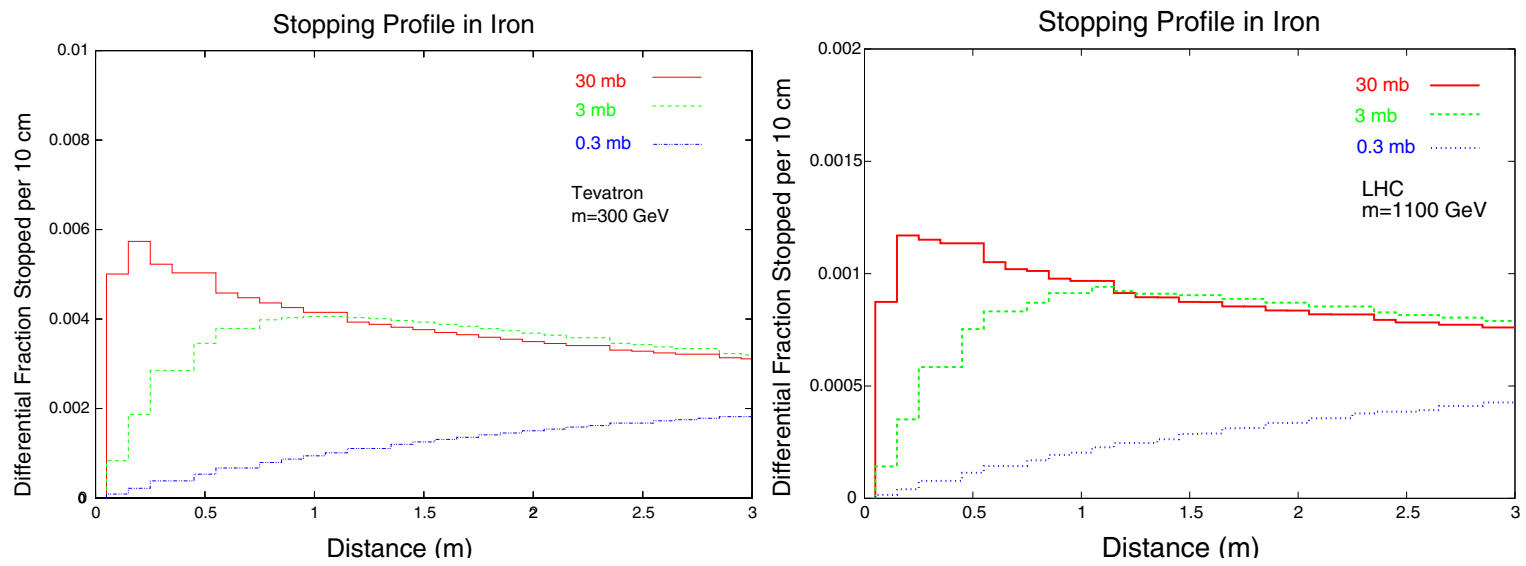

Figure 5: The fraction of gluinos stopped per $10 \mathrm{~cm}$ as a function of distance in the case where the isosinglet hadron is the lightest (Mass Region 1). There are three curves in each plot representing different cross sections for R-meson to R-baryon conversion. The different colors represent different cross-sections for meson to baryon conversion $\left(\sigma v_{r e l}=30 \mathrm{mb}\right.$ (solid blue), $\sigma v_{\text {rel }}=3 \mathrm{mb}$ (dashed red), $\sigma v_{\text {rel }}=0.3 \mathrm{mb}$ (dotted green)). The left plot is for the Tevatron and gluino mass of $300 \mathrm{GeV}$ while the right plot is for the LHC and a mass of 1100 $\mathrm{GeV}$.

\subsection{Late Decays in Detectors}

A stopped gluino will decay into either a pair of jets and electroweak-ino or a single jet and an electroweak-ino. These jets originate where the gluino stopped and will mostly be in the densest regions of the detector, the electromagnetic and hadronic calorimeters. The jets will be pointed in any direction. When the decay of the gluino is reconstructed under the assumption that the event came from the beam interaction point (as it would be initially), 
it would appear grossly unbalanced. There would only be activity in the side of the detector where the gluino decays. Therefore, we expect the decays to pass the level-one trigger for missing transverse energy.

The response of the calorimeters to jets that are not pointed back to the interaction region is complicated and detector dependent. If the jets are completely contained in a single cell or tower, this may look like a "hot cell" or "spike." A typical $100 \mathrm{GeV}$ jet contains about 30 particles, so it seems likely that there will be some leakage into other towers or cells. It is beyond the scope of this paper to attempt to calculate how much leakage there is in each detector, but clearly having several adjacent cells or towers with energy deposition will help eliminate hardware related backgrounds.

The decay of the gluino happens long after the beam crossing that produced it and the decay will be uncorrelated with any beam crossing. At Run II at the Tevatron, the beam crossings are every 396 ns. Due to the relatively long spacing between bunch crossings at Run II, at CDF 132 ns is recorded around any given bunch crossing. This means that one third of the decaying gluinos have a chance to be recorded. For the ones that are recorded, most of the decays will be significantly out-of-time, appearing before or after a beam crossing. This may help reduce QCD backgrounds which appear within $10 \mathrm{~ns}$ of the beam crossing. At the LHC, the bunch crossings occur every $25 \mathrm{~ns}$ and the calorimeters are always recording. This means that any gluino decay can be recorded. However, the closeness of the bunch spacings means that gluino decays may not be identified as out-of-time and can not be differentiated from QCD backgrounds by timing information.

Many gluinos are stopped deep in the hadronic calorimeter and will not deposit any energy in electromagnetic calorimeter or in the tracking chamber. In the case where the jet is completely contained, the signal is particularly clean. The hadronic calorimeter "lights-up" with hundreds of GeV of energy, but without any activity in any other portion of the detector. High energy QCD jets typically have dozens of charged particles and also deposit energy into the EM calorimeter and leave tracks. This seems to indicate that the background from SM physics is controllable, and the backgrounds seem to be dominated by detector backgrounds.

Backgrounds from cosmic rays are a serious consideration since they occur out-of-time like a late-decaying gluino. Most high energy cosmic rays are muons because they can effectively penetrate the atmosphere and shielding. While a gluino decays into jets that deposit their energy inside the calorimeter, a cosmic ray muon typically transverses the detector without depositing much of its energy. Most cosmic rays will also appear in the muon chamber, so vetoing muon tracks could be a useful discriminant. Neutral hadronic cosmic rays can not be vetoed in this way and are a potential background. However, they do not penetrate matter effectively and when they do reach the detector, they interact with the outermost layers of the hadronic calorimeter.

Another unique feature of the signal is that the gluinos that stop are predominantly central and axially symmetric. In Fig. 6, we illustrate the density of stopped gluinos in the ATLAS calorimeters. The density of stopped gluinos can be up to $30 /(10 \mathrm{~cm})^{3}$ in the central region. This distribution distinguishes late decaying gluinos from both jet backgrounds (uniformly distributed in rapidity) and cosmic rays (uniform throughout the detector and not axially symmetric). Depending on the total number of gluinos stopped, it might be possible to do sideband subtraction to eliminate QCD and cosmic ray backgrounds.

A stopped gluino can decay into two jets plus a neutralino $\left(\tilde{g} \rightarrow q \bar{q} \chi^{0}\right)$, or a single jet plus 


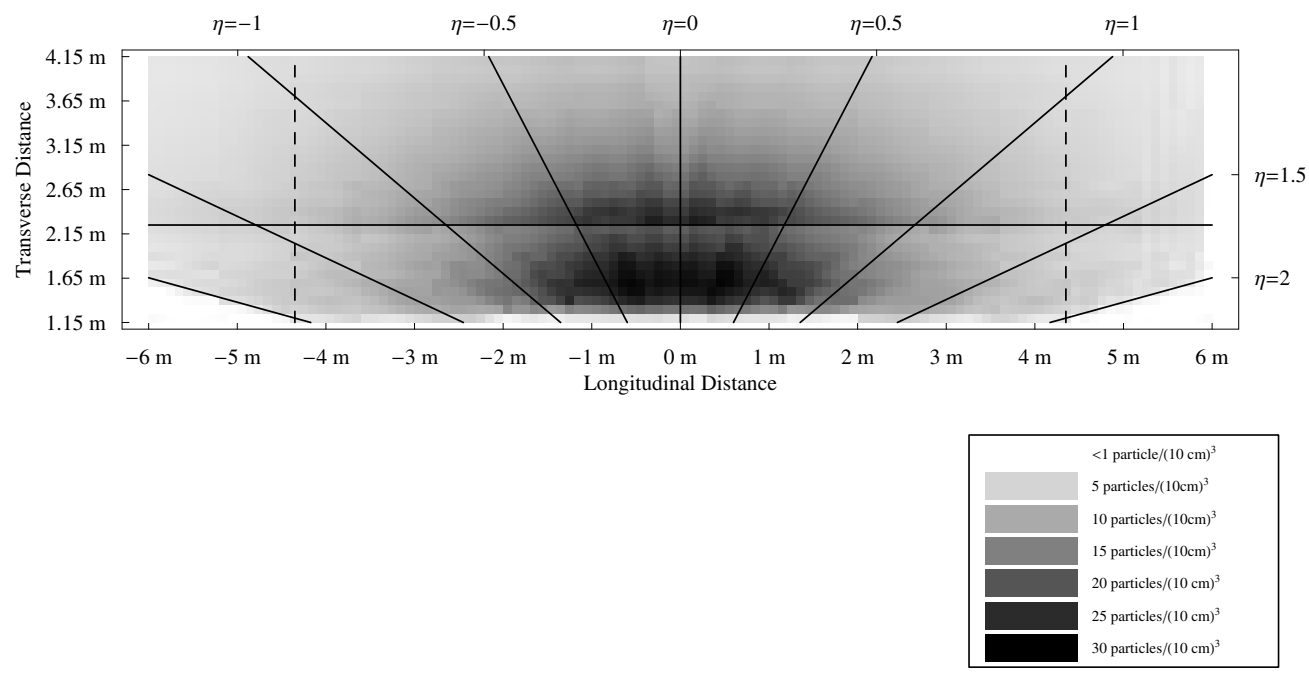

Figure 6: The density of stopped gluinos in the ATLAS detector for $m_{\tilde{g}}=300 \mathrm{GeV}$ at the LHC, assuming a $100 \mathrm{fb}^{-1}$ of data. Note the increased density of stopped gluinos at low pseudo-rapidity. The vertical lines represent the start of the end cap calorimeter.

a neutralino $\left(\tilde{g} \rightarrow g \chi^{0}\right)$. The relative branching fraction is sensitive to the gluino mass and the scale of supersymmetry breaking[15, 12, 23]. The two-body branching fraction increases as the gluino mass decreases, or as the scale of SUSY breaking increases. It would be of interest to study the extent to which the two jets originating within the calorimeter might be disentangled from one another. This requires a detailed understanding of the response of the detector to jets propagating from within the calorimeter and is beyond the scope of this work. If the two jets can be distinguished, this could be an important handle on distinguishing these events from background. Even more optimistically, one might get some rough handle on the branching ratio of the two-body versus three body decays, thereby indirectly determining the SUSY breaking scale.

Another possibility is to use the decays of the gluinos into the non-LSP electroweak-inos to search for their discovery ${ }^{2}$. For instance, the decay $\tilde{g} \rightarrow \chi_{2}^{0} j \rightarrow \chi_{1}^{0} \mu^{+} \mu^{-} j$ will have two energetic muons leaving the hadronic calorimeter from the point where the jet deposited its energy. This may be a potential discovery channel for electroweak-inos at the LHC.

\section{Discussion}

Split supersymmetry presents an experimental challenge. As in the supersymmetric standard model (SSM), only colored particles are abundantly produced at hadronic colliders. However, unlike the SSM, split SUSY does not allow the study of new electroweak particles through cascade decays of colored particles - only the gluino is colored, and it decays outside of the

\footnotetext{
${ }^{2}$ We thank Lian-Tao Wang for bringing this to our attention.
} 
inner detector. Since electroweak particles are difficult to produce directly, discovering split SUSY hinges on the identification of the gluino. There are now four distinct possibilities to discovering the long-lived gluino at the Tevatron and the LHC.

- One possibility is by measuring an excess of monojets through a $\tilde{g} \tilde{g} j$ event[5]. Gluinos will leave very little energy in the detector, so the jet will look unbalanced. However, a variety of new physics can lead to a monojet signature. To claim discovery of split SUSY at the Tevatron or LHC will require additional signals beyond monojets. It might be possible to distinguish the split supersymmetry monojet signature from other monojet events [5]. It is possible that the gluino might deposit small puffs $(\sim 1 \mathrm{GeV})$ of additional energy as it traverses the calorimeter through hadronic interactions. This would earmark the new physics as strongly interacting.

- The second approach is to search for anomalously slow particles in the tracking chambers. Outside the calorimeters most of the R-hadrons will be R-baryons and therefore half will be charged. In [6], there is a proposal for using the muon chamber to search for charged R-hadrons and found a discovery potential for ATLAS up to $1700 \mathrm{GeV} . d E / d x$ measurements can also be used in the inner tracking chamber and look for anomalously heavy charged particles [24], Reference [5] estimated that the Tevatron has a reach of $430 \mathrm{GeV}$ for $2 \mathrm{fb}^{-1}$ if the all R-hadrons are charged and a reach of $2.4 \mathrm{TeV}$ at the LHC for $100 \mathrm{fb}^{-1}$. Unfortunately, in Mass Region 1, the reach of this strategy is reduced since almost all of the R-hadrons will be neutral in the tracking chamber. In this case, only a fraction of the R-baryons will likely be charged, and the reach will probably be much closer to $200 \mathrm{GeV}$ for the Tevatron and $1.2 \mathrm{TeV}$ for the LHC.

- Another possibility for discovering the gluino is a search for charge oscillating events, known as "flippers." (see, e.g., [11]) Flippers appear experimentally difficult. In tracking chambers, where charge oscillation can best be measured, there is not much material to stimulate the hadronic interactions that lead to the effect.

- Seeing a stopped gluino decay is the last known discovery channel for the gluino. Only a fraction of the gluinos actually stop, so this hurts the reach of this method. It may take a very careful observation of these events to be sure that these are not a fluctuation of a QCD event or a cosmic ray. However, this approach would allow us to infer the existence of a particle with a finite lifetime.

Ideally, some combination of these measurements might lead to a convincing discovery of split supersymmetry.

Since we made several assumptions about strong cross-sections and spectroscopy, we would like to comment on the robustness of the conclusions derived. The most robust method of stopping was a two step process: R-mesons converted to R-baryons which then stopped via ionization losses. The second part of this process is extremely robust, and relies only on the well-established physics of the Bethe-Bloch equation. The first part is only slightly more uncertain-it hinges on a reasonable sized ( $\gtrsim 1 / v_{\text {rel }} \mathrm{mb}$ ) cross section for the meson to baryon conversion. If this process were not exothermic, these conclusions would not hold. This seems unlikely given the lightness of the pion. Then, gluinos would only 
stop if the isotriplet mesons are produced, either because they are the lightest (Mass Region 3 ), or by charge exchange (Mass Region 2). The other possible loophole is if the ( $\tilde{g} u d s$ ) state is so light that other baryons weakly decay to it on time scales faster than the stopping time. Then meson to baryon conversion only succeeds in creating ( $\tilde{g} u d s$ ) hadrons, which do not lose their energy efficiently. In this case, stopping gluinos would again rely on having a light charged meson. However, given the mass cost additional strange quark, we view such a spectroscopy as unlikely, and consider the conclusions here to be robust.

We close with a few additional very speculative comments on determining the gluino lifetime. While the method described here would indicate the decay of the particle with a lifetime greater than its stopping time (100 ns), the inability to associate the decay of the gluino with the time of its production prevents an accurate measurement. If enough gluinos are produced then it might be possible to look for decays immediately after the accelerator is shutdown to make an estimate of the gluino's lifetime. If the gluino is lighter than 500 $\mathrm{GeV}$, then it is possible for the gluino to live up to $10^{5}$ years. These may never decay inside the detector while it is running. Still the R-hadrons will be caught inside the detector. A search looking for exotic heavy nuclei could be done in principle, using the material of the detector. In principle, these exotic gluino-containing nuclei might even decay at a later time, allowing one to probe lifetimes exceeding the duration of the LHC running.

\section{Acknowledgments}

We thank N. Arkani-Hamed for collaboration at an early stage of this work. We acknowledge H. Frisch and D. Toback for discussions of calorimeter response and timing at CDF, K. Einsweiller, E. Paganis, and G. Polesello for discussions on triggering, calorimetery and timing at ATLAS, and D. B. Kaplan, A. Nelson, and M. Savage for discussions on nuclear capture. We thank D. Amidei, L. Dixon, E. Katz, A. Kraan, B. Lillie, M. Peskin, J. Qian, T. Rizzo, L.-T. Wang, and J.Wells for many useful conversations.

\section{References}

[1] N. Arkani-Hamed and S. Dimopoulos, arXiv:hep-th/0405159.

[2] G. F. Giudice and A. Romanino, Nucl. Phys. B 699, 65 (2004) [Erratum-ibid. B 706, 65 (2005)] [arXiv:hep-ph/0406088]. N. Arkani-Hamed, S. Dimopoulos, G. F. Giudice and A. Romanino, Nucl. Phys. B 709, 3 (2005) [arXiv:hep-ph/0409232].

[3] J. D. Wells, arXiv:hep-ph/0306127. J. D. Wells, Phys. Rev. D 71, 015013 (2005) [arXiv:hep-ph/0411041].

[4] W. Kilian, T. Plehn, P. Richardson and E. Schmidt, Eur. Phys. J. C 39, 229 (2005) [arXiv:hep-ph/0408088].

[5] J. L. Hewett, B. Lillie, M. Masip and T. G. Rizzo, JHEP 0409, 070 (2004) [arXiv:hep$\mathrm{ph} / 0408248]$. 
[6] A. C. Kraan, Eur. Phys. J. C 37, 91 (2004) [arXiv:hep-ex/0404001]. A. C. Kraan, arXiv:hep-ex/0506009.

[7] K. Cheung and W. Y. Keung, Phys. Rev. D 71, 015015 (2005) [arXiv:hep-ph/0408335].

[8] G. R. Farrar, Phys. Rev. Lett. 76, 4111 (1996) [arXiv:hep-ph/9603271]. S. Raby, Phys. Lett. B 422, 158 (1998) [arXiv:hep-ph/9712254]. A. Mafi and S. Raby, Phys. Rev. D 62, 035003 (2000) [arXiv:hep-ph/9912436].

[9] H. Baer, K. m. Cheung and J. F. Gunion, Phys. Rev. D 59, 075002 (1999) [arXiv:hep$\mathrm{ph} / 9806361]$.

[10] A. Heister et al. [ALEPH Collaboration], Eur. Phys. J. C 31, 327 (2003) [arXiv:hepex/0305071].

[11] R. Culbertson et al. [SUSY Working Group Collaboration], arXiv:hep-ph/0008070.

[12] M. Toharia and J. D. Wells, arXiv:hep-ph/0503175.

[13] L. Anchordoqui, H. Goldberg and C. Nunez, Phys. Rev. D 71, 065014 (2005) [arXiv:hep$\mathrm{ph} / 0408284]$;

J. G. Gonzalez, S. Reucroft and J. Swain, arXiv:hep-ph/0504260.

[14] K. Hamaguchi, Y. Kuno, T. Nakaya and M. M. Nojiri, Phys. Rev. D 70, 115007 (2004) [arXiv:hep-ph/0409248];

J. L. Feng and B. T. Smith, Phys. Rev. D 71, 015004 (2005) [Erratum-ibid. D 71, 0109904 (2005)] [arXiv:hep-ph/0409278].

[15] A. Arvanitaki, C. Davis, P. W. Graham, A. Pierce and J. G. Wacker, arXiv:hep$\mathrm{ph} / 0504210$.

[16] S. J. Brodsky, J. F. Gunion and D. E. Soper, Phys. Rev. D 36, 2710 (1987).

[17] H. L. Lai et al., Phys. Rev. D 55, 1280 (1997) [arXiv:hep-ph/9606399];

H. L. Lai and W. K. Tung, Z. Phys. C 74, 463 (1997) [arXiv:hep-ph/9701256].

[18] E. Eichten, I. Hinchliffe, K. D. Lane and C. Quigg, Rev. Mod. Phys. 56, 579 (1984) [Addendum-ibid. 58, 1065 (1986)].

[19] M. Spira, arXiv:hep-ph/9711408. W. Beenakker, R. Hopker, M. Spira and P. M. Zerwas, Nucl. Phys. B 492, 51 (1997) [arXiv:hep-ph/9610490].

[20] J. Sabin and J. Oddershede, Phys Rev. A 39 (1989) 1033.

L.E. Porter, Nucl. Inst. Methods B 69 (1992) 39.

C. J. Bakker and E. Segre, Phys. Rev. 81 (1951) 489.

[21] E. Fermi and E. Teller, Phys. Rev. 72, 399 (1947).

[22] J. Lindhard, M. Scharff and E. M. Sciott, Kgl. Danske Vikdenskab. Mat. Fys. Medd. 344 (1964).

J. Lindhard, M. Scharff, Phys. Rev. 124 (1961) 128. 
[23] P. Gambino, G. F. Giudice and P. Slavich, arXiv:hep-ph/0506214.

[24] F. Abe et al. [CDF Collaboration], Phys. Rev. D 46, 1889 (1992). 